SLAC-PUB-8184

SU-ITP-99/32

hep-th/9906239

May 6, 2021

\title{
One-loop corrections to the D3 brane action.
}

\author{
Marina Shmakova \\ Stanford Linear Accelerator Center, \\ Stanford University, Stanford, CA 94309; \\ University of Tennessee, Knoxville, TN 37996.
}

\begin{abstract}
$\underline{\text { ABSTRACT }}$
We calculate one-loop corrections to the effective Lagrangian for the D3 brane. We perform the gauge-fixing of the $\kappa$-symmetric Born-Infeld D3 brane action in the flat background using Killing gauge. The linearized supersymmetry of the gaugefixed action coincides with that of the $\mathcal{N}=4$ Yang-Mills theory. We use the helicity amplitude and unitarity technique to calculate the one-loop amplitudes at order $\alpha^{4}$. The counterterms and the finite 1-loop corrections are of the form $(\partial F)^{4}$ and their supersymmetric generalization. This is to be contrasted with the Born-Infeld action which contains $(F)^{4}$ and other terms which do not depend on derivatives of the vector field strength.
\end{abstract}

e-mail: shmakova@slac.stanford.edu. 


\section{Introduction}

The action for supersymmetric D3-branes in flat and curved type IIB supergravity backgrounds has been studied extensively during the last few years [1, 2, 3, 国. In addition to extended supersymmetry these actions have a local $\kappa$-symmetry and half of the 32 fermionic fields can be "gauged away" by fixing the $\kappa$-gauge. Moreover, of the 32 supersymmetries of type IIB supergravity, 16 are realized linearly and 16 non-linearly. The interpretation of all these supersymmetries is closely related to the problem of the correspondence between supersymmetric $\mathcal{N}=4 U(N)$-symmetric Yang-Mills theories and D3-brane Born-Infeld-type $U(1)$-symmetric actions [4]. The $\mathcal{N}=4$ Yang-Mills theory in $\mathrm{d}=4$ is not only renormalizable but even finite [5]. The D3 brane action has been considered so far only as an effective action as it is not renormalizable by power counting in $\mathrm{d}=4$. Still, there are $16+16$ linear and non-linear global symmetries in the gauge-fixed action (or equivalently a local $\kappa$-symmetry of the classical action). Is it possible that these symmetries are strong enough to forbid the counterterms in the D3 brane action which have a higher number of derivatives than the classical Born-Infeld action? One has to take into account that the D3 brane action has terms of the form $\left(\left(F_{\mu \nu}\right)^{n}+\ldots\right)$ however, terms of the form $(\partial F)^{n}$ or $\left(\partial^{2} F\right)^{n}$ are not present.

As a first step in resolving these issues, in particular to clarify the relation between the effective action of the open string theory, Yang-Mills theory and effective action of the D3-brane theory, we will treat the $\kappa$-symmetric action of the D3-brane on the same footing as supergravity in $\mathrm{d}=4$ or supersymmetric Yang-Mills theory in $d>4$. A priori these theories are not renormalizable. However a careful analysis of supersymmetric higher derivative counterterms was performed in the past, as well as some actual calculations [6].

In this paper we will present one-loop corrections to the D3-brane Abelian BornInfeld action in a flat background, and we will show that there are ultraviolet divergences, in contrast to the finite $\mathrm{d}=4 \mathcal{N}=4$ Super-Yang-Mills [5], and that the form of the couterterm is $\left(s^{2}+t^{2}+u^{2}\right) F^{4} \sim(\partial F)^{4}$. To fix the $\kappa$-symmetry we will employ the so-called Killing gauge used in [7] for gauge-fixing of the GS type IIB string in 
the $\mathrm{AdS}_{5} \times \mathrm{S}^{5}$ background. In this gauge we will be able to directly identify the linear supersymmetries of the gauge-fixed D3 brane action with the YM supersymmetries. We will compare this to the symmetries of the D3 brane action in $\theta_{1}=0$ gauge, proposed in [3]. The calculation of the tree amplitudes and one-loop corrections gives the same results in the other gauge, of course. However, in the Killing gauge the lowest order Lagrangian contains only quartic interaction terms while in $\theta_{1}=0$ gauge there are cubic as well as quartic interactions. Therefore it is easier to perform one-loop calculations in the Killing gauge.

It is interesting that the terms with the $(\partial F)^{4}$ structure were found in the effective action for the open superstrings in the paper by Andreev and Tseytlin [8] from the string S-matrix term $\left(s^{2}+t^{2}+u^{2}\right) F^{4}$. This kind of terms were also found in the more recent papers by Hashimoto and Klebanov [9] in which they computed the scattering amplitude for massless vectors on a D-brane in string theory. In the low energy limit their result also contain the term proportional to $\left(s^{2}+t^{2}+u^{2}\right) F^{4}$. The appearance of this structure in our calculations and in the string effective action could mean that the supersymmetry fixes this structure in the unique way. This would also mean that the local $\kappa$-symmetry after gauge fixing corresponds to the global $\mathcal{N}=4$ supersymmetry at least in this approximation.

To perform one-loop calculations we will use the helicity amplitude method that has been developed extensively in the last 10 years (for reviews see [10, 11]). The main ideas of this method that we will employ here are the spinor helicity representation for polarization vectors [12], supersymmetry identities [13] and unitarity cuts [14]. This method has been used for loop calculations in Yang-Mills theory and recently also in gravity [15]. It turns out that it is extremely useful for the D3 brane calculations.

In Sec. 2 we will discuss the D3-brane action and find the SUSY transformations in the Killing gauge. In Sec. 3 the $\mathcal{N}=4$ supersymmetric Lagrangian of the BornInfeld theory will be presented up to the quartic order in the fields. In Sec. 4 we will apply the helicity amplitude formalism to the D3-brane action. We will present tree and one-loop amplitudes for the quartic Lagrangian in the Killing gauge and give the expressions for the one-loop corrections and counterterms in this theory. In Sec. 5 
we will discuss the form of the Lagrangian up to the quartic order in $\theta_{1}=0$ gauge and Sec. 6 is a conclusion.

\section{The D3 brane action in a flat background}

In this paper we will closely follow the notations of [4]. The supersymmetric D3brane action is a sum of the Born-Infeld (BI) and Wess-Zumino (WZ) terms [1, 3, 4] that depend on superspace coordinates $X^{M}=\left(x^{\hat{a}}, \Theta^{I}\right)$ and an Abelian world-volume gauge field strength $d A$

$$
S=S_{B I}+S_{W Z}
$$

where the Born-Infeld part is

$$
S_{B I}=-T_{3} \int_{M_{4}} d^{4} \sigma \sqrt{-\operatorname{det}\left(G_{i j}+2 \pi \alpha^{\prime} \mathcal{F}_{i j}\right)}
$$

where $T_{3} \sim \frac{1}{\left(2 \pi \alpha^{\prime}\right)^{2}}$ is the D3-brane tension [16] and $2 \pi \alpha^{\prime}$ is the inverse string tension. $G_{i j}$ is the pullback to the $\mathrm{d}=4$ world-volume metric of the $\mathrm{d}=10$ Minkowski metric $\eta^{\hat{a} \hat{b}}=(-+\ldots+)$ with $i, j=0 \ldots 3 ; \hat{a}, \hat{b}=0 \ldots 9$ :

$$
G_{i j}=L_{i}^{\hat{a}} \eta^{\hat{a} \hat{b}} L_{i}^{\hat{b}}, \quad L^{\hat{a}}(X(\sigma))=d \sigma^{i} L_{i}^{\hat{a}}
$$

and in the flat background the supervielbeins are

$$
\begin{aligned}
L^{\mathcal{I}} & =d \Theta^{\mathcal{I}}, \quad \mathcal{I}=1,2 \\
L^{\hat{a}} & =d x^{\hat{a}}-i \bar{\Theta}^{\mathcal{I}} \Gamma^{\hat{a}} d \Theta^{\mathcal{I}} .
\end{aligned}
$$

The field strength $F=\frac{1}{2} \mathcal{F}_{i j} d \sigma^{i} \wedge d \sigma^{j}$ is the supersymmetric extension of $d A$ :

$$
F=d A+2 \mathrm{i} \int_{0}^{1} d t L_{t}^{\hat{a}} \wedge \bar{\Theta} \eta^{a b} \Gamma^{\hat{b}} \mathcal{K} L_{t}
$$

The WZ part of the action is

$$
S_{W Z}=2 \mathrm{i} T_{3} \int_{M_{4}} \int_{0}^{1} d t\left(\frac{1}{6} \bar{\Theta} \widehat{L}_{t} \wedge \widehat{L}_{t} \wedge \widehat{L}_{t} \mathcal{E} L_{t}+2 \pi \alpha^{\prime} \bar{\Theta} \widehat{L}_{t} \wedge \mathrm{F}_{t} \wedge \mathcal{J} L_{t}\right)+T_{3} \int_{M_{4}} \Omega_{4}^{(\text {bos })}
$$


where $\widehat{L}=L^{\hat{a}} \Gamma^{\hat{a}}$ and $L_{t}^{\hat{a}}(x, \Theta) \equiv L^{\hat{a}}(x, t \Theta), \quad L_{t}(x, \Theta) \equiv L(x, t \Theta)$. The $\Theta$ independent part of the 4 -form in the flat background is $\Omega_{4}^{(b o s)}=d^{4} \sigma$. The $\Gamma^{\hat{a}}$ are the $\mathrm{d}=10$ Dirac matrices and the matrices $\mathcal{E}, \mathcal{J}, \mathcal{K}$ in (6) and (5) act on the $\mathrm{SO}(2)$ indices of type IIB spinors $\Theta^{\mathcal{I}}$ :

$$
\mathcal{E}=\left(\begin{array}{cc}
0 & 1 \\
-1 & 0
\end{array}\right), \quad \mathcal{J}=\left(\begin{array}{ll}
0 & 1 \\
1 & 0
\end{array}\right), \quad \mathcal{K}=\left(\begin{array}{cc}
1 & 0 \\
0 & -1
\end{array}\right)
$$

The supersymmetry transformations with global parameter $\varepsilon$ and $\kappa$-symmetry transformations with local parameter $\kappa(\sigma)$ for this action are [1, 田

$$
\begin{aligned}
\delta_{\varepsilon} \Theta^{\mathcal{I}} & =\varepsilon^{\mathcal{I}} \\
\delta_{\varepsilon} x^{\hat{a}} & =-\mathrm{i}\left(\bar{\Theta} \Gamma^{\hat{a}} \varepsilon\right) \\
\delta_{\varepsilon} A & =\mathrm{i} d x^{\hat{a}} \bar{\Theta} \Gamma^{\hat{a}} \mathcal{K} \varepsilon-\frac{1}{6}\left(d \bar{\Theta} \Gamma_{\hat{a}} \Theta\right)\left(\bar{\Theta} \Gamma^{\hat{a}} \mathcal{K} \varepsilon\right)-\frac{1}{6}\left(d \bar{\Theta} \Gamma_{\hat{a}} \mathcal{K} \Theta\right)\left(\bar{\Theta} \Gamma^{\hat{a}} \varepsilon\right) \\
\delta_{\kappa} \Theta^{\mathcal{I}} & =\kappa^{\mathcal{I}} \\
\delta_{\kappa} x^{\hat{a}} & =\mathrm{i}\left(\bar{\Theta} \Gamma^{\hat{a}} \kappa\right) \\
\delta_{\kappa} A & =-\mathrm{i} d x^{\hat{a}} \bar{\Theta} \Gamma^{\hat{a}} \mathcal{K} \kappa+\frac{1}{2}\left(d \bar{\Theta} \Gamma_{\hat{a}} \Theta\right)\left(\bar{\Theta} \Gamma^{\hat{a}} \mathcal{K} \kappa\right)+\frac{1}{2}\left(d \bar{\Theta} \Gamma_{\hat{a}} \mathcal{K} \Theta\right)\left(\bar{\Theta} \Gamma^{\hat{a}} \kappa\right),
\end{aligned}
$$

where the SUSY parameter $\varepsilon^{\mathcal{I}}$ is a constant type IIB Majorana-Weyl spinor. Half of the components of the $\kappa$-transformation parameter are projected out by the condition

$$
\kappa=\frac{1}{2}(1+\Gamma) \kappa, \quad \Gamma^{2}=1
$$

with

$$
\Gamma=\left(\begin{array}{cc}
0 & \zeta \\
\tilde{\zeta} & 0
\end{array}\right)=\frac{\epsilon^{i_{1} \ldots i_{4}}\left(\frac{1}{4 !} \Gamma_{i_{1} \ldots i_{4}} \mathcal{E}+\frac{2 \pi \alpha^{\prime}}{4} \Gamma_{i_{1} i_{2}} \mathcal{F}_{i_{3} i_{4}} \mathcal{J}+\frac{\left(2 \pi \alpha^{\prime}\right)^{2}}{8} \mathcal{F}_{i_{1} i_{2}} \mathcal{F}_{i_{3} i_{4}} \mathcal{E}\right)}{\sqrt{-\operatorname{det}\left(G_{i j}+2 \pi \alpha^{\prime} \mathcal{F}_{i j}\right)}}
$$

where $\Gamma_{i_{1} \ldots i_{n}} \equiv \widehat{L}_{\left[i_{1}\right.} \ldots \widehat{L}_{\left.i_{n}\right]}$.

Following [7, 17] we will choose the "Killing gauge" for the $\kappa$-symmetry. In [0] $\Theta^{1,2}$ were choose to be the Killing spinors of the $A d S_{5}$ background, for the flat background we can simply introduce new variables $\vartheta^{ \pm}$

$$
\vartheta^{ \pm}=U \Theta^{1,2} \Rightarrow\left(\begin{array}{c}
\vartheta^{+} \\
\vartheta^{-}
\end{array}\right)=\frac{1}{\sqrt{2}}\left(\begin{array}{cc}
1 & \Gamma^{(4)} \\
1 & -\Gamma^{(4)}
\end{array}\right)\left(\begin{array}{c}
\Theta^{1} \\
\Theta^{2}
\end{array}\right)
$$


where $\Gamma^{(4)}=\Gamma^{0} \Gamma^{1} \Gamma^{2} \Gamma^{3}$ with

$$
\begin{aligned}
& \Gamma^{p}=\gamma^{p} \otimes 1, \quad p=0, \ldots, 3 \\
& \Gamma^{t}=\gamma^{5} \otimes \tilde{\gamma}^{t}, \quad t=4, \ldots, 9
\end{aligned}
$$

Here $\gamma^{p}$, and $\gamma^{5}$ are standard Dirac matrices with $\left\{\gamma^{p}, \gamma^{p^{\prime}}\right\}=2 \eta^{p p^{\prime}}$ and $\tilde{\gamma}^{t}$ are $8 \times 8$ six-dimensional Dirac matrices with $\left\{\tilde{\gamma}^{t}, \tilde{\gamma}^{t^{\prime}}\right\}=2 \delta^{t t^{\prime}}$.

The supersymmetry and $\kappa$-symmetry transformations (10) and (13) with the new parameters $\varepsilon^{ \pm}=U \varepsilon^{\mathcal{I}}, \kappa^{ \pm}=U \kappa^{\mathcal{I}}$ are

$$
\left(\begin{array}{c}
\delta_{\kappa+\varepsilon} \vartheta^{+} \\
\delta_{\kappa+\varepsilon} \vartheta^{-}
\end{array}\right)=\left(\begin{array}{c}
\varepsilon^{+} \\
\varepsilon^{-}
\end{array}\right)+\frac{1}{2}\left(\begin{array}{cc}
1-\frac{1}{2} C & \frac{1}{2} A \\
-\frac{1}{2} A & 1+\frac{1}{2} C
\end{array}\right)\left(\begin{array}{c}
\kappa^{+} \\
\kappa^{-}
\end{array}\right) .
$$

Here the matrices $A$ and $C$ are

$$
A=\zeta \Gamma^{(4)}+\Gamma^{(4)} \tilde{\zeta}, \quad C=\zeta \Gamma^{(4)}-\Gamma^{(4)} \tilde{\zeta}
$$

and explicit expressions for $\zeta$ and $\tilde{\zeta}$ are given in (15).

We gauge away half of the fermionic coordinates by imposing $\vartheta^{-}=0$. This condition also requires that $\delta_{\kappa+\varepsilon} \vartheta^{-}=0$. Gauge fixing $\kappa$-symmetry by the condition $\kappa^{+}=0$ we get:

$$
\begin{aligned}
& \delta_{\kappa+\varepsilon} \vartheta^{+}=\frac{1}{4} A \kappa^{-}+\varepsilon^{+} \\
& \delta_{\kappa+\varepsilon} \vartheta^{-}=\frac{1}{2}\left(1+\frac{1}{2} C\right) \kappa^{-}+\varepsilon^{-}=0 .
\end{aligned}
$$

Eq. (21) gives a relation between $\kappa^{-}$and $\varepsilon^{-}$,

$$
\kappa^{-}=-2\left(1+\frac{1}{2} C\right)^{-1} \varepsilon^{-} .
$$

Finally we get

$$
\delta_{\kappa+\varepsilon} \vartheta^{+}=\frac{1-\zeta \Gamma^{(4)}}{1+\zeta \Gamma^{(4)}} \varepsilon^{-}+\varepsilon^{+} .
$$

Using this transformation on $A^{p}$ and $x^{\hat{a}}=x^{p}, y^{t}(p=0, \cdots, 3 ; t=4, \cdots, 9)$ :

$$
\begin{aligned}
\delta_{\kappa+\varepsilon} x^{p} & =i \bar{\vartheta}^{+} \Gamma^{p}\left(\tau \varepsilon^{-}-\varepsilon^{+}\right) \\
\delta_{\kappa+\varepsilon} y^{t} & =-2 i \bar{\vartheta}^{+} \Gamma^{t} \varepsilon^{-} \\
\delta_{\kappa+\varepsilon} A^{p} & =2 i \bar{\vartheta}^{+} \Gamma^{p} \varepsilon^{-}-\frac{2}{3} \partial_{p} \bar{\vartheta}^{+} \Gamma_{a} \vartheta^{+} \bar{\vartheta}^{+} \Gamma^{a} \varepsilon^{-}-i \partial_{p} y^{t} \bar{\vartheta}^{+} \Gamma^{t}\left(\tau \varepsilon^{-}-\varepsilon^{+}\right)
\end{aligned}
$$


where

$$
\tau=\frac{1-\zeta \Gamma^{(4)}}{1+\zeta \Gamma^{(4)}} .
$$

The additional general coordinate reparametrization is necessary to satisfy the static gauge $\left(x^{p}=\sigma^{p}\right)$ condition $\delta x^{p}=0$

$$
\begin{aligned}
\delta_{\xi} \vartheta & =\xi^{p} \partial_{p} \vartheta \\
\delta_{\xi} x^{\hat{a}} & =\xi^{p} \partial_{p} x^{\hat{a}}
\end{aligned}
$$

then from $\delta_{\kappa+\varepsilon+\xi} x^{p}=0$ and $\partial_{i} x^{p}=\delta_{i}^{p}$ it follows that $\xi^{i}=-i \bar{\vartheta}^{+} \Gamma^{i}\left(\tau \varepsilon^{-}-\varepsilon^{+}\right)$. Finally the SUSY-transformations for remaining nonzero fermions, scalars and vectors are:

$$
\begin{aligned}
\delta_{\kappa+\varepsilon+\xi} \vartheta^{+} & =\left(\tau \varepsilon^{-}+\varepsilon^{+}\right)+\xi^{i} \partial_{i} \vartheta^{+} \\
\delta_{\kappa+\varepsilon+\xi} y^{t} & =-2 i \bar{\vartheta}^{+} \Gamma^{t} \varepsilon^{-}+\xi^{i} \partial_{i} y^{t} \\
\delta_{\kappa+\varepsilon+\xi} A^{i} & =2 i \bar{\vartheta}^{+} \Gamma^{i} \varepsilon^{-}-\frac{2}{3} \partial_{i} \bar{\vartheta}^{+} \Gamma_{a} \vartheta^{+} \bar{\vartheta}^{+} \Gamma^{a} \varepsilon^{-} \\
& -i \partial_{i} y^{t} \bar{\vartheta}^{+} \Gamma^{t}\left(\tau \varepsilon^{-}-\varepsilon^{+}\right)+\left(\xi^{j} \partial_{j} A^{i}+\partial_{i} \xi^{j} A^{j}\right)
\end{aligned}
$$

It is easy to see that the transformations for $y^{t}$ are linear and do not contain an $\varepsilon^{+}$ part.

From here on we will drop the "+" in the spinor notation i.e. $\vartheta^{+} \Rightarrow \vartheta$. In the new variables the supervierbeins (4) are:

$$
L=d \vartheta ; \quad L^{p}=d x^{p}-i \bar{\vartheta} \Gamma^{p} d \vartheta ; \quad L^{t}=d y^{t}
$$

\section{The Quartic $\mathcal{N}=4$ Lagrangian}

The D3-brane BI action (2) simplifies considerably in the Killing gauge. In this case

$$
\begin{aligned}
G_{i j}+2 \pi \alpha^{\prime} \mathcal{F}_{i j} & =\eta^{i j}-i \bar{\vartheta} \Gamma_{i} \partial_{j} \vartheta-i \bar{\vartheta} \Gamma_{j} \partial_{i} \vartheta-\bar{\vartheta} \Gamma_{p} \partial_{i} \vartheta \bar{\vartheta} \Gamma_{p} \partial_{j} \vartheta+\partial_{i} y^{t} \partial_{i} y^{t} \\
& +2 \pi \alpha^{\prime}\left(\mathrm{F}_{i j}-i \partial_{i} y^{t} \bar{\vartheta} \Gamma_{p} \partial_{j} \vartheta+i \partial_{j} y^{t} \bar{\vartheta} \Gamma_{t} \partial_{i} \vartheta\right)
\end{aligned}
$$

where $\mathrm{F}_{i j}=\partial_{i} A_{j}-\partial_{j} A_{i}$ and the WZ-term is

$$
L_{W Z}=-T_{3}\left(i \bar{\theta} \not \partial \theta-\frac{i}{2} \partial_{l} \bar{\theta} \Gamma^{j k l} \Gamma^{t} \Gamma^{t^{\prime}} \theta \partial_{j} y^{t} \partial_{k} y^{t^{\prime}}+\frac{1}{2}\left((\bar{\theta} \not \partial \theta)^{2}-\bar{\theta} \Gamma^{i} \partial_{j} \theta \bar{\theta} \Gamma^{j} \partial_{i} \theta\right)\right.
$$




$$
\begin{aligned}
& -\frac{1}{4} \epsilon^{i j k l} \partial_{k} \bar{\theta} \Gamma^{c} \theta \partial_{l} \bar{\theta} \Gamma_{c} \Gamma^{(4)} \Gamma^{t} \Gamma^{t^{\prime}} \theta \partial_{i} y^{t} \partial_{j} y^{t^{\prime}}-\frac{1}{2} \epsilon^{i j k l} \partial_{k} \bar{\theta} \Gamma^{t} \Gamma^{(4)} \theta \partial_{l} \bar{\theta} \Gamma^{t^{\prime}} \theta \partial_{i} y^{t} \partial_{j} y^{t^{\prime}} \\
& +\frac{i}{6} \epsilon^{i j k l} \epsilon_{a b c l} \bar{\theta} \Gamma^{a} \partial_{i} \theta \bar{\theta} \Gamma^{b} \partial_{j} \theta \bar{\theta} \Gamma^{c} \partial_{k} \theta+\frac{1}{24} \epsilon^{i j k l} \epsilon_{a b c d} \bar{\theta} \Gamma^{a} \partial_{i} \theta \bar{\theta} \Gamma^{b} \partial_{j} \theta \bar{\theta} \Gamma^{c} \partial_{k} \theta \bar{\theta} \Gamma^{d} \partial_{l} \theta \\
& \left.-\left(2 \pi \alpha^{\prime}\right) \frac{i}{2} \partial_{l} \bar{\theta} \Gamma^{i j k l} \mathrm{~F}_{i j} \Gamma^{t} \theta \partial_{k} y^{t}\right)
\end{aligned}
$$

Using a series expansion for $\sqrt{\operatorname{det}\left(G_{i j}+2 \pi \alpha^{\prime} \mathcal{F}_{i j}\right)}$ and a standard redefinition of the fields we get the effective Lagrangian up to the fourth order in fields (and coupling constant $\left.\alpha=2 \pi \alpha^{\prime}\right)$ :

$$
\begin{aligned}
L_{B I+W Z}^{4} & =2 i \bar{\theta} \not \partial \theta+\frac{1}{4} \mathrm{~F}_{i j} \mathrm{~F}_{j i}-\frac{1}{2} \partial_{i} y^{t} \partial_{i} y^{t}+\alpha^{2}\left((\bar{\theta} \not \partial \theta)^{2}-\bar{\theta} \Gamma^{i} \partial_{j} \theta \bar{\theta} \Gamma^{j} \partial_{i} \theta\right) \\
& +\frac{\alpha^{2}}{8}\left(\mathrm{~F}_{i j} \mathrm{~F}^{j k} \mathrm{~F}_{k l} \mathrm{~F}^{l i}-\frac{1}{4}\left(\mathrm{~F}_{i j} \mathrm{~F}_{j i}\right)^{2}\right)+\frac{\alpha^{2}}{4}\left(\partial_{i} y^{t} \partial_{j} y^{t} \partial_{i} y^{t^{\prime}} \partial_{j} y^{t^{\prime}}-\frac{1}{2}\left(\partial_{i} y^{t} \partial_{i} y^{t}\right)^{2}\right) \\
& -\frac{\alpha^{2}}{2}\left(\partial_{i} y^{t} \partial_{j} y^{t} \mathrm{~F}_{i k} \mathrm{~F}^{k j}-\frac{1}{4} \partial_{i} y^{t} \partial_{i} y^{t} \mathrm{~F}_{j k} \mathrm{~F}^{k j}\right) \\
& -i \alpha^{2}\left(\left(\bar{\theta} \Gamma^{i} \partial_{j} \theta\right)\left(\partial_{i} y^{t} \partial_{j} y^{t}\right)-\frac{1}{2}(\bar{\theta} \not \partial \theta)\left(\partial_{i} y^{t} \partial_{i} y^{t}\right)+\frac{1}{2} \partial_{k} \bar{\theta} \Gamma^{i j k} \partial_{i} y^{t} \Gamma^{t} \partial_{j} y^{t^{\prime}} \Gamma^{t^{\prime}} \theta\right) \\
& +i \alpha^{2}\left(\bar{\theta} \Gamma^{i} \partial_{j} \theta \mathrm{F}^{j k} \mathrm{~F}_{k i}-\frac{1}{4} \bar{\theta} \not \partial \theta \mathrm{F}^{i j} \mathrm{~F}_{j i}\right) \\
& +i \alpha^{2}\left(\bar{\theta} \partial_{i} y^{t} \Gamma^{t} \partial_{j} \theta \mathrm{F}_{i j}-\frac{1}{2} \bar{\theta} \partial_{i} y^{t} \Gamma^{t} \Gamma^{(4)} \partial_{l} \theta \mathrm{F}_{j k} \epsilon^{i j k l}\right)
\end{aligned}
$$

To establish the analogy between this theory and $\mathcal{N}=4$ super-Yang-Mills theory it is helpful to use the chiral representation for $\tilde{\gamma}^{t}$ (see Appendix) where the $\mathrm{SU}(4)$ symmetry is manifest. Using this representation we will introduce new notations for the scalar fields $s^{I J}=\frac{1}{2}\left(\tilde{\sigma}_{t}^{-1}\right)^{I J} y^{t}$ with $\mathrm{SU}(4)$ indices $I, J=1,2,3,4$. The 16component spinor $\theta$ will be represented as four $\mathrm{d}=4$ Majorana spinors,

$$
\theta=\frac{\tilde{\psi}^{(I)}}{2}=\left(\begin{array}{c}
\lambda_{\alpha I} \\
\bar{\lambda}^{\dot{\alpha} I}
\end{array}\right),
$$

where the extra factor 2 was introduced for convenience and the $\mathrm{d}=4$ chiral projections are

$$
\tilde{\psi}_{I}^{+}=\frac{1}{2}\left(1+\gamma^{5}\right) \tilde{\psi}^{(I)} ; \quad \tilde{\psi}^{-I}=\frac{1}{2}\left(1-\gamma^{5}\right) \tilde{\psi}^{(I)} .
$$

The quartic $\mathcal{N}=4$ Lagrangian in terms of the new 4-dim YM variables $\left(g, \tilde{\psi}^{I}, s^{I J}\right)$ with $g^{i}=A^{i}$ is

$$
L_{B I+W Z}^{4}=i \frac{1}{2} \overline{\tilde{\psi}}_{I} \not \partial \tilde{\psi}^{I}+\frac{1}{4} \mathrm{~F}_{i j} \mathrm{~F}_{j i}-\frac{1}{2} \partial_{i} s^{I J} \partial_{i} s_{I J}
$$




$$
\begin{aligned}
& +\frac{\alpha^{2}}{16}\left(\left(\overline{\tilde{\psi}}_{I} \not \partial \tilde{\psi}^{I}\right)^{2}-\overline{\tilde{\psi}}_{I} \gamma^{i} \partial_{j} \tilde{\psi}^{I} \overline{\tilde{\psi}}_{J} \gamma^{j} \partial_{i} \tilde{\psi}^{J}\right) \\
& +\frac{\alpha^{2}}{8}\left(\mathrm{~F}_{i j} \mathrm{~F}^{j k} \mathrm{~F}_{k l} \mathrm{~F}^{l i}-\frac{1}{4}\left(\mathrm{~F}_{i j} \mathrm{~F}_{j i}\right)^{2}\right)+\frac{\alpha^{2}}{4}\left(\partial_{i} s^{I J} \partial_{j} s_{I J} \partial_{i} s^{I^{\prime} J^{\prime}} \partial_{j} s_{I^{\prime} J^{\prime}}-\frac{1}{2}\left(\partial_{i} s^{I J} \partial_{i} s_{I J}\right)^{2}\right) \\
& -\frac{\alpha^{2}}{2}\left(\partial_{i} s^{I J} \partial_{j} s_{I J} \mathrm{~F}_{i k} \mathrm{~F}^{k j}-\frac{1}{4} \partial_{i} s^{I J} \partial_{i} s_{I J} \mathrm{~F}_{j k} \mathrm{~F}^{k j}\right) \\
& -\frac{i \alpha^{2}}{4}\left(\left(\overline{\tilde{\psi}}_{I} \gamma^{i} \partial_{j} \tilde{\psi}^{I}\right)\left(\partial_{i} s^{I J} \partial_{j} s_{I J}\right)-\frac{1}{2}\left(\overline{\tilde{\psi}}_{I} \not \partial \tilde{\psi}^{I}\right)\left(\partial_{i} s^{I J} \partial_{i} s_{I J}\right)\right. \\
& \left.-2 \partial_{k} \overline{\tilde{\psi}}_{I} \gamma^{i j k} \partial_{i} s^{I J} \partial_{j} s_{J K} \tilde{\psi}^{K}\right) \\
& +\frac{i \alpha^{2}}{4}\left(\overline{\tilde{\psi}}_{I} \gamma^{i} \partial_{j} \tilde{\psi}^{I} \mathrm{~F}^{j k} \mathrm{~F}_{k i}-\frac{1}{4} \overline{\tilde{\psi}}_{I} \not \partial \tilde{\psi}^{I} \mathrm{~F}^{i j} \mathrm{~F}_{j i}\right) \\
& +\frac{i \alpha^{2}}{2}\left(\overline{\tilde{\psi}}_{I}^{+} \partial_{i} s^{I J} \gamma^{5} \partial_{j} \tilde{\psi}_{J}^{+} \mathrm{F}_{i j}-\overline{\tilde{\psi}}^{I-} \partial_{i} s^{I J} \gamma^{5} \partial_{j} \tilde{\psi}^{J-} \mathrm{F}_{i j}\right. \\
& \left.+\frac{1}{2} \overline{\tilde{\psi}}_{I}^{+} \gamma^{i j k l} \partial_{i} s^{I J} \gamma^{5} \partial_{l} \tilde{\psi}_{J}^{+} \mathrm{F}_{j k}-\frac{1}{2} \overline{\tilde{\psi}}_{I}^{-} \gamma^{i j k l} \partial_{i} s^{I J} \gamma^{5} \partial_{l} \tilde{\psi}_{J}^{-} \mathrm{F}_{j k}\right) .
\end{aligned}
$$

where $\gamma^{i_{1} \cdots i_{k}} \equiv \frac{1}{k !} \gamma^{\left[i_{1}\right.} \cdots \gamma^{\left.i_{k}\right]}$. The parameter $\tau$ from Eq. 27) is, up to linear order in the fields:

$$
\tau_{I J} \approx \frac{1}{2} \not \partial y^{t} \Gamma_{I J}^{t}-\frac{1}{4} \gamma^{i j} \mathrm{~F}_{i j} \delta_{I J}
$$

The supersymmetry transformation now looks like:

$$
\begin{aligned}
\delta_{\text {susy }} \tilde{\psi}^{(I)} & =\left(\begin{array}{cc}
-\frac{1}{2} \gamma^{i j} \mathrm{~F}_{i j} \delta_{I J} & 2 \not \partial \gamma^{5} s_{I J} \\
-2 \not \partial \gamma^{5} s^{I J} & -\frac{1}{2} \gamma^{i j} \mathrm{~F}_{i j} \delta_{I J}
\end{array}\right) \cdot\left(\begin{array}{c}
\varepsilon_{J}^{-} \\
\bar{\varepsilon}^{-J}
\end{array}\right) \\
\delta_{\text {susy }} g^{i} & =i \overline{\tilde{\psi}} \gamma^{i} \varepsilon^{-} \\
\delta_{\text {susy }} s_{I J} & =i \varepsilon^{-}{ }_{I} \tilde{\psi}_{J}-i \varepsilon^{-}{ }_{J} \tilde{\psi}_{I}+i \bar{\varepsilon}_{K}^{-} \overline{\tilde{\psi}}_{L} \epsilon^{I J K L} .
\end{aligned}
$$

Finally, using a chiral representation for $\gamma^{i}$ we get

$$
\begin{aligned}
\delta_{\text {susy }} \tilde{\psi}_{I} & =-\frac{1}{2} \sigma^{i j} \mathrm{~F}_{i j} \varepsilon^{-}{ }_{I}-2 \sigma^{i} \partial_{i} s_{I J} \bar{\varepsilon}^{-J} \\
\delta_{\text {susy }} g^{i} & =-i \varepsilon_{I}^{-} \sigma^{i} \overline{\tilde{\psi}}_{I}+i \tilde{\psi}^{I} \sigma^{i}\left(\bar{\varepsilon}^{-}\right)^{I} \\
\delta_{\text {susy }} s_{I J} & =i \varepsilon^{-}{ }_{I} \tilde{\psi}_{J}-i \varepsilon^{-}{ }_{J} \tilde{\psi}_{I}+i \bar{\varepsilon}_{K} \overline{\tilde{\psi}}_{L} \epsilon^{I J K L}
\end{aligned}
$$

which is exactly the $\mathcal{N}=4$ transformation given in 18 . 


\section{Helicity amplitudes.}

To calculate one loop corrections to the D3-brane action we use the helicity amplitude technique (see for example the review paper [10] and references therein). In the spinor helicity formalism, positive- and negative-helicity massless spinors $\psi$ are represented as

$$
|p \pm\rangle=\psi_{ \pm}(p)=\frac{1}{2}\left(1 \pm \gamma^{5}\right) \psi(p)
$$

with antisymmetric spinor products

$$
\begin{aligned}
\left\langle p_{i}-\mid p_{j}+\right\rangle & \equiv\left\langle p_{i} p_{j}\right\rangle \equiv\langle i j\rangle \\
\left\langle p_{i}+\mid p_{j}-\right\rangle & \equiv\left[p_{i} p_{j}\right] \equiv[i j] \\
\langle i j\rangle[j i] & =2 p_{i} \cdot p_{j} \equiv s_{i j} .
\end{aligned}
$$

Negative- and positive-helicity polarization vectors also can be represented through spinors $|p \pm\rangle$

$$
\varepsilon_{\mu}^{+}(p ; k)=\frac{\left\langle k-\left|\gamma_{\mu}\right| p-\right\rangle}{\sqrt{2}\langle k p\rangle}, \quad \varepsilon_{\mu}^{-}(p ; k)=-\frac{\left\langle k+\left|\gamma_{\mu}\right| p+\right\rangle}{\sqrt{2}[k p]},
$$

where $k_{\mu}\left(k^{2}=0\right)$ is a "reference" momentum which corresponds to the particular choice of gauge for the external legs. Due to the gauge invariance of the amplitudes, the vector $k$ drops out of the final expressions. The supersymmetry transformations (41),(42) and (43) can also be rewritten in terms of bosonic states with definite helicity $g^{ \pm}(p)=\varepsilon_{\mu}^{ \pm}(p) g^{\mu}$ and spinors $\tilde{\psi}_{ \pm}(p)=|p \pm\rangle$ as [13]

$$
\begin{aligned}
{\left[Q_{I}(p), \tilde{\psi}_{J}^{ \pm}(k)\right] } & =\mp \Gamma^{\mp}(k, p) g^{ \pm}(k) \delta_{I J} \mp \Gamma^{ \pm}(k, p) s_{I J}^{ \pm}, \\
{\left[Q_{I}(p), g^{ \pm}(k)\right] } & =\mp i \Gamma^{ \pm}(k, p) \tilde{\psi}_{I}^{ \pm}, \\
{\left[Q_{I}(p), s_{J K}^{ \pm}(k)\right] } & = \pm i \Gamma^{\mp} \delta^{I J} \tilde{\psi}_{K}^{ \pm} \mp i \Gamma^{\mp} \delta^{I K} \tilde{\psi}_{J}^{ \pm} \pm i \Gamma^{ \pm} \tilde{\psi}_{L}^{ \pm} \epsilon^{I J K L},
\end{aligned}
$$

where $\Gamma^{+}(k, p)=\bar{\eta}[p k], \quad \Gamma^{-}(k, p)=\eta\langle p k\rangle$, and $\eta$ is a numerical Grassmann parameter.

Using the Lagrangian (39) it is easy to find the tree-level helicity amplitudes. For the vector bosons we have:

$$
\begin{aligned}
4 !\left(\mathrm{F}_{i j} \mathrm{~F}^{j k} \mathrm{~F}_{k l} \mathrm{~F}^{l i}-\frac{1}{4}\left(\mathrm{~F}_{i j} \mathrm{~F}_{j i}\right)^{2}\right) & =\left(t_{8}\right)_{\mu_{1} \nu_{1} \mu_{2} \nu_{2} \mu_{3} \nu_{3} \mu_{4} \nu_{4}} F_{\mu_{1} \nu_{1}} F_{\mu_{2} \nu_{2}} F_{\mu_{3} \nu_{3}} F_{\mu_{4} \nu_{4}} \\
& =16\left(t_{8}\right)_{\mu_{1} \nu_{1} \cdots \mu_{4} \nu_{4}} k_{1}^{\mu_{1}} k_{2}^{\mu_{2}} k_{3}^{\mu_{3}} k_{4}^{\mu_{4}} \epsilon_{1}^{\nu_{1}} \epsilon_{2}^{\nu_{2}} \epsilon_{3}^{\nu_{3}} \epsilon_{4}^{\nu_{4}}
\end{aligned}
$$


where $\left(t_{8}\right)$ is given in eq.(9.A.19) in 19

$$
\begin{aligned}
\left(t_{8}\right)_{\mu_{1} \nu_{1} \mu_{2} \nu_{2} \mu_{3} \nu_{3} \mu_{4} \nu_{4}} & =\left(-\frac{1}{2}\left(\delta_{\mu_{1} \mu_{2}} \delta_{\nu_{1} \nu_{2}}-\delta_{\mu_{1} \nu_{2}} \delta_{\nu_{1} \mu_{2}}\right)\left(\delta_{\mu_{3} \mu_{4}} \delta_{\nu_{3} \nu_{4}}-\delta_{\mu_{3} \nu_{4}} \delta_{\nu_{3} \mu_{4}}\right)\right. \\
& \left.+\frac{1}{2}\left(\delta_{\nu_{1} \mu_{2}} \delta_{\nu_{2} \mu_{3}} \delta_{\nu_{3} \mu_{4}} \delta_{\nu_{4} \mu_{1}}+\text { antisym. of }\left[\mu_{\mathrm{i}} \nu_{\mathrm{i}}\right]\right)\right) \\
& +((1324)+(1342) \text { permutations })
\end{aligned}
$$

and $k_{i}$ and $\epsilon_{i}$ are the momentum and polarization vectors for an external vector boson leg.

In the YM theory with non-Abelian gauge group $\mathrm{SU}\left(N_{c}\right)$ the tree amplitudes are usually represented according to color decomposition [10, 20] as a sum of partial subamplitudes with fixed cyclic ordering of external legs multiplied by the color factors:

$$
\mathcal{A}_{n}^{\text {tree }}(1,2, \cdots n)=\sum_{\sigma \in \frac{S_{n}}{Z_{n}}} \operatorname{Tr}\left(T^{a_{\sigma(1)}} T^{a_{\sigma(2)}} \cdots T^{a_{\sigma(n)}}\right) A_{n}^{\text {tree }}(\sigma(1), \sigma(2), \cdots \sigma(n))
$$

where $\frac{S_{n}}{Z_{n}}$ is the set of all non-cyclic permutations and $T^{a_{\sigma(n)}}$ are matrices of the fundamental representation of the $\mathrm{SU}\left(N_{c}\right)$ color group. In the D3-brane case the gauge group is an Abelian U(1) and color factors will be simply reduced to 1 .

All four-particle tree amplitudes are proportional to $\alpha^{2}(\sqrt{39})$ and to simplify the notations we will let $\alpha^{2}=1$. Due to the supersymmetric Ward identities (SWI) 13 the only nonzero four vector-boson tree amplitude in this case is $A_{4}^{\text {tree }}\left(g_{1}^{-} g_{2}^{-} g_{3}^{+} g_{4}^{+}\right)$ (with two - and two + helicity bosons)

$$
\begin{aligned}
A_{4}^{\text {tree }}\left(1^{-}, 2^{-}, 3^{+}, 4^{+}\right) & =-i \frac{4 !}{8}\left\langle 3,4\left|\left(\mathrm{~F}_{i j} \mathrm{~F}_{j k} \mathrm{~F}_{k l} \mathrm{~F}_{l i}-\frac{1}{4}\left(\mathrm{~F}_{i j} \mathrm{~F}_{j i}\right)^{2}\right)\right| 1,2\right\rangle \\
\text { 2 } 2 \mathcal{Q}^{\gamma} & =-i \frac{4}{2}\left(t_{8}\right)_{\mu_{1} \nu_{1} \mu_{2} \nu_{2} \mu_{3} \nu_{3} \mu_{4} \nu_{4}} k_{1}^{\mu_{1}} k_{2}^{\mu_{2}} k_{3}^{\mu_{3}} k_{4}^{\mu_{4}} \epsilon_{1}^{\nu_{1}} \epsilon_{2}^{\nu_{2}} \epsilon_{3}^{\nu_{3}} \epsilon_{4}^{\nu_{4}} \\
و^{2} \partial_{2} & =\frac{i}{2} \frac{s t\langle 12\rangle^{4}}{\langle 12\rangle\langle 23\rangle\langle 34\rangle\langle 41\rangle}
\end{aligned}
$$

where $s=s_{12}, t=s_{14}, u=s_{13}$ are Mandelstam variables. This amplitude is related to the standard $\mathcal{N}=4 \mathrm{SYM}$ four-gluon color-ordered sub-amplitude [10] by:

$$
A_{4}^{\text {tree }}\left(1^{-}, 2^{-}, 3^{+}, 4^{+}\right)=\frac{1}{2} \text { st } A_{4}^{\text {tree }, Y M}(1,2,3,4) .
$$


It is important to notice that the RHS of (56) is totally symmetric under external leg permutations [13].

The nonzero vector-scalar tree amplitudes are:

$$
\begin{aligned}
A_{4}^{\text {tree }}\left(g^{-},\right. & \left.s_{I J}^{-}, s_{I J}^{+}, g^{+}\right)= \\
g_{G_{6}^{-}} & =-i \frac{4}{2}\left(t_{8}\right)_{\mu_{1} t \mu_{2} t \mu_{3} \nu_{3} \mu_{4} \nu_{4}} k_{1}^{\mu_{1}} k_{2}^{\mu_{2}} k_{3}^{\mu_{3}} k_{4}^{\mu_{4}} \epsilon_{3}^{\nu_{3}} \epsilon_{4}^{\nu_{4}} \\
s_{I J}^{\prime} S_{\sigma_{I J}} & =\frac{i}{2}\langle 12\rangle\langle 13\rangle[43][42] \\
& =\frac{s t\langle 12\rangle^{2}\langle 13\rangle^{2}}{2\langle 12\rangle^{4}} A_{4}^{\text {tree,YM}(1,2,3,4) .}
\end{aligned}
$$

There are two kinds of four-scalar amplitudes:

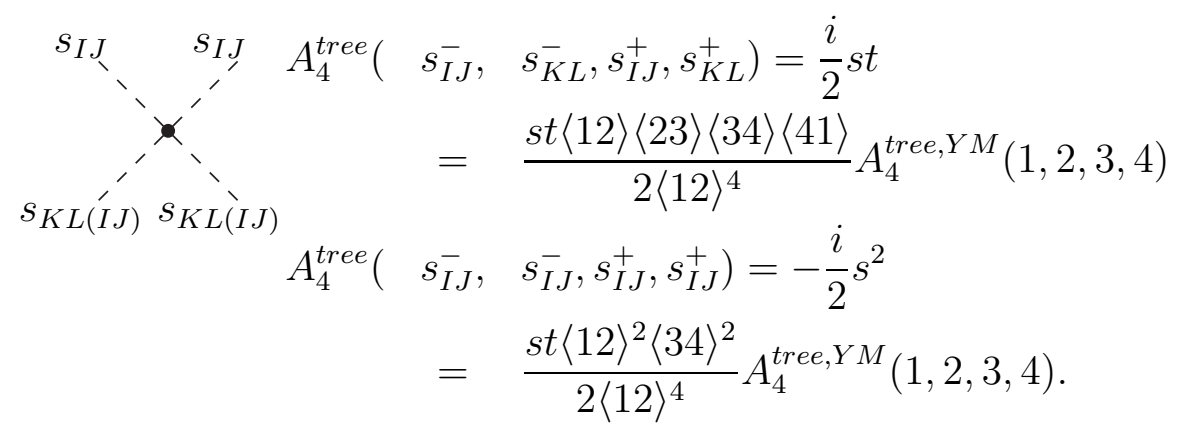

The four fermion tree amplitudes include two nonzero ones:

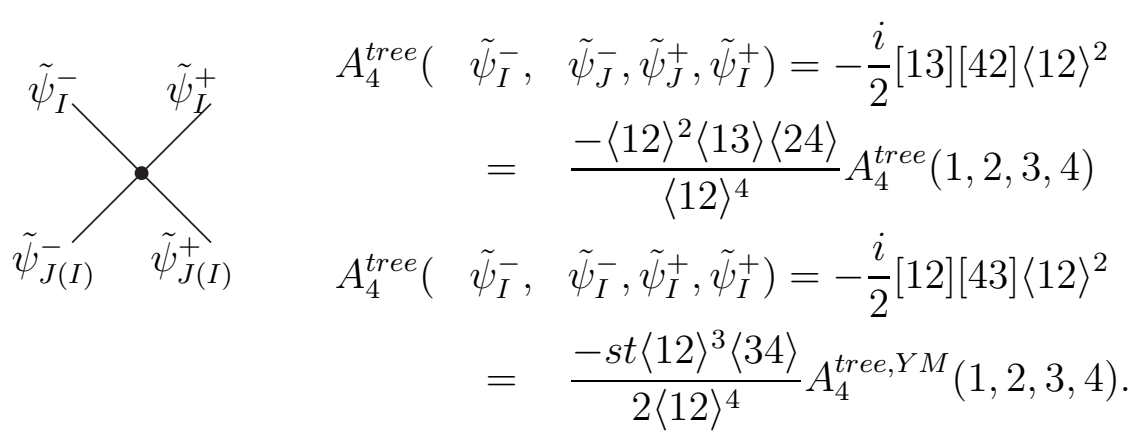

The next set of diagrams contains a mixture of two fermions and two bosons. There are five nonzero tree amplitudes: 

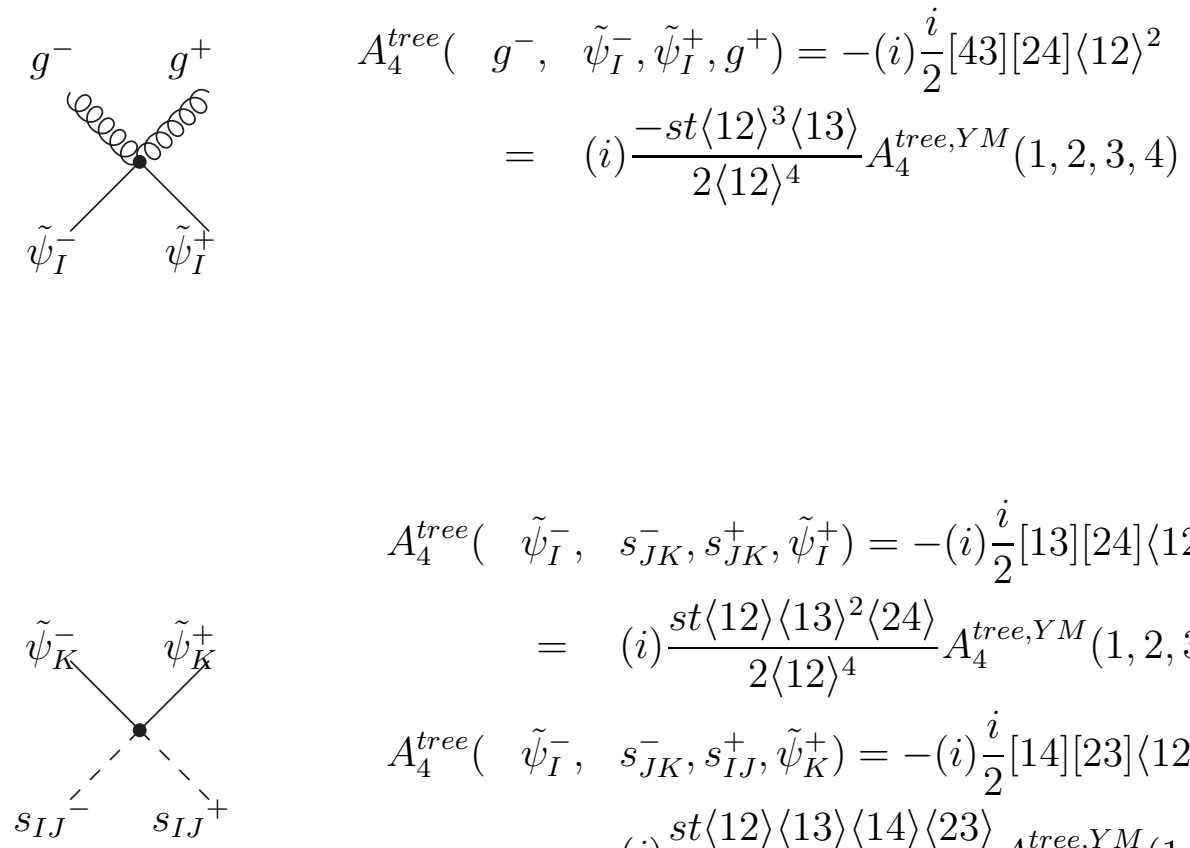

$$
\begin{aligned}
A_{4}^{\text {tree }}( & \left.\tilde{\psi}_{I}^{-}, \quad s_{J K}^{-}, s_{J K}^{+}, \tilde{\psi}_{I}^{+}\right)=-(i) \frac{i}{2}[13][24]\langle 12\rangle\langle 13\rangle \\
= & (i) \frac{s t\langle 12\rangle\langle 13\rangle^{2}\langle 24\rangle}{2\langle 12\rangle^{4}} A_{4}^{\text {tree }, Y M}(1,2,3,4) \\
A_{4}^{\text {tree }}\left(\tilde{\psi}_{I}^{-},\right. & \left.s_{J K}^{-}, s_{I J}^{+}, \tilde{\psi}_{K}^{+}\right)=-(i) \frac{i}{2}[14][23]\langle 12\rangle\langle 13\rangle \\
= & (i) \frac{s t\langle 12\rangle\langle 13\rangle\langle 14\rangle\langle 23\rangle}{2\langle 12\rangle^{4}} A_{4}^{\text {tree }, Y M}(1,2,3,4)
\end{aligned}
$$

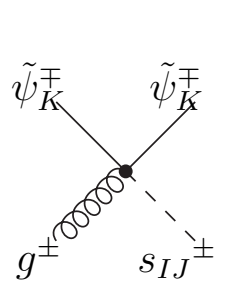

$$
\begin{aligned}
& A_{4}^{\text {tree }}\left(\tilde{\psi}_{I}^{-}, \quad \tilde{\psi}_{J}^{-}, s_{I J}^{+}, g^{+}\right)= \\
& =i \frac{s t\langle 12\rangle^{2}\langle 13\rangle\langle 23\rangle}{2\langle 12\rangle^{4}} A_{4}^{\text {tree }, Y M}(1,2,3,4) \\
& A_{4}^{\text {tree }}\left(g^{-}, \quad s_{I J}^{-}, \tilde{\psi}_{I}^{+}, \tilde{\psi}_{J}^{+}\right)= \\
& =-i \frac{s t\langle 12\rangle^{2}\langle 13\rangle\langle 14\rangle}{2\langle 12\rangle^{4}} A_{4}^{\text {tree }, Y M}(1,2,3,4)
\end{aligned}
$$

This set of tree amplitudes has exactly the same relative factors between them as for $\mathcal{N}=4$ SUSY Yang-Mills theory given in [15] (extra $i$ 's come from difference between metrics signature which are $g^{\mu \nu}=(+---)$ in 15 and $g^{\mu \nu}=(-+++)$ in this paper ). The general expression connecting SYM amplitudes and the D3 brane 
amplitudes is:

$$
A_{4}^{\text {tree }}\left(l_{1}, l_{2}, l_{3}, l_{4}\right)=\frac{s t}{2} A_{4}^{\text {tree }, Y M}\left(l_{1}, l_{2}, l_{3}, l_{4}\right)
$$

where $l_{1}, l_{2}, l_{3}, l_{4}$ are the momenta of the particles from $\mathrm{N}=4$ SUSY multiplet.

The main difference between the standard $\mathcal{N}=4 \mathrm{SYM}$ and the D3-brane effective theory (39) is that the first one is finite in $d=4$. The following calculation shows that the D3-brane action is not one-loop finite in the flat background.

A very useful relation for one-loop calculations in the standard $\mathcal{N}=4 \mathrm{SYM}$ theory is [15, 21]:

$$
\begin{aligned}
\sum_{S_{1}, S_{2} \in(N=4)} & A_{4}^{\text {tree }, Y M}\left(-l_{1}^{S_{1}}, 1,2, l_{2}^{S_{2}}\right) \times A_{4}^{\text {tree }, Y M}\left(-l_{2}^{S_{2}}, 3,4, l_{1}^{S_{1}}\right) \\
= & \frac{-i s t A_{4}^{\text {tree }, Y M}(1,2,3,4)}{\left(l_{1}-k_{1}\right)^{2}\left(l_{2}-k_{3}\right)^{2}}
\end{aligned}
$$

where the sum is over all particles in the $\mathcal{N}=4$ multiplet and 1,2,3,4 stands for the momenta of the external particles. This statement is true for any dimension and its proof can be found in [21, 22]. For our case (with relation (68)) it becomes:

$$
\begin{aligned}
\sum_{S_{1}, S_{2} \in(N=4)} & A_{4}^{\text {tree }(D 3)}\left(-l_{1}^{S_{1}}, 1,2, l_{2}^{S_{2}}\right) \times A_{4}^{\text {tree }(D 3)}\left(-l_{2}^{S_{2}}, 3,4, l_{1}^{S_{1}}\right) \\
= & \frac{-i s^{2}}{4} \operatorname{st} A_{4}^{\text {tree }, Y M}(1,2,3,4) \\
= & \frac{-i s^{2}}{2} A_{4}^{\text {tree }(D 3)}(1,2,3,4)
\end{aligned}
$$

here $A_{4}^{\text {tree(D3) }}(1,2,3,4)$ is the four vector-boson D3-brane amplitude (56).

The $\mathcal{N}=4$ supersymmetry of the D3-brane action allows us to use a unitaritybased construction of the one loop amplitudes, as it has done in $\mathcal{N}=4$ SuperYang-Mills theory (see review [11] and references therein). In this technique, one obtains the imaginary part of the one-loop amplitude from the product of the tree amplitudes, and then reconstructs the real part up to a possible polynomial function. The good ultraviolet behavior of $\mathcal{N}=4$ super-Yang-Mills theory makes it possible to reconstruct the whole amplitude without the additive polynomial ambiguity. The one-loop amplitudes that can be reconstructed that way ("cut-constructible") must 
satisfy a certain loop-momentum "power-counting" criterion given, for example, in [11.

One-loop amplitudes in the standard $\mathcal{N}=4$ SYM theory obtained by using the cut-reconstruction formalism can be expressed as:

$$
\begin{aligned}
A_{4}^{N=4,1-\text { loop }}(1,2,3,4) & =i g^{2} s_{12} s_{23} A_{4}^{\text {tree }}(1,2,3,4)\left(C_{1234} I_{4}^{1-\text { loop }}\left(s_{12}, s_{23}\right)\right. \\
& \left.+C_{3124} I_{4}^{1-l o o p}\left(s_{12}, s_{13}\right)+C_{2314} I_{4}^{1-\text { loop }}\left(s_{23}, s_{13}\right)\right)
\end{aligned}
$$

where $C_{1234}$ is a color factor for the non-Abelian gauge group and $I_{4}^{1-l o o p}$ is a one-loop four-point (box) integral,

$$
I_{4}^{1-\text { loop }}\left(s_{12} s_{23}\right)=\int \frac{d^{D} p}{(2 \pi)^{D}} \frac{1}{p^{2}\left(p-k_{1}\right)^{2}\left(p-k_{1}-k_{2}\right)^{2}\left(p+k_{4}\right)^{2}} .
$$

The expression (69) was used to simplify the direct product of the tree amplitudes.

The expressions (69) and (70) for $\mathcal{N}=4$ amplitudes are correct in all dimensions and can be used [15, 23] to reconstruct complete massless loop amplitudes. The D3brane effective Lagrangian (39) in the Killing gauge does not contain three-particle vertices, hence only bubble diagrams will contribute to the one-loop corrections to the four-particle amplitudes. In this case the expression for one-loop corrections considerably simplifies and can be written as:

$$
\begin{aligned}
& A_{4}^{D 3,1-\text { loop }}(1,2,3,4)= \\
= & \frac{i \alpha^{4}}{4} s_{12} s_{23} A_{4}^{\text {tree }, Y M}(1,2,3,4)\left(s_{12}^{2} I_{2}^{1-\text { loop }}\left(s_{12}\right)+s_{13}^{2} I_{2}^{1-l o o p}\left(s_{13}\right)+s_{14}^{2} I_{2}^{1-l o o p}\left(s_{14}\right)\right) \\
= & \frac{i \alpha^{4}}{2} A_{4}^{\text {tree }(D 3)}(1,2,3,4)\left(s^{2} I_{2}^{1-l o o p}(s)+t^{2} I_{2}^{1-l o o p}(t)+u^{2} I_{2}^{1-l o o p}(u)\right) .
\end{aligned}
$$

Here we have taken into account that $s_{12} s_{23} A_{4}^{\text {tree,YM}}(1,2,3,4)$ from (56) is symmetric under momenta permutations and color factors are gone because gauge field in (5) is an Abelian one. Instead of the box integrals (72) expression (73) contains only two-point one-loop integrals:

$$
I_{2}^{1-\text { loop }}\left(s_{12}\right)=\int \frac{d^{4-2 \epsilon} p}{(2 \pi)^{4-2 \epsilon}} \frac{1}{p^{2}\left(p-k_{1}-k_{2}\right)^{2}} .
$$

This integral is ultra-violet divergent and in dimensional reduction it has the form:

$$
I_{2}^{1-l o o p}(s)=i(4 \pi)^{\epsilon-2} \frac{\Gamma(1+\epsilon) \Gamma^{2}(1-\epsilon)}{\epsilon(1-2 \epsilon) \Gamma(1-2 \epsilon)}\left(\frac{-s}{\mu^{2}}\right)^{-\epsilon}
$$




$$
\sim \frac{i}{(4 \pi)^{2}} \frac{1}{\epsilon}\left[1+\epsilon\left(2-\gamma-\log \frac{-s}{\mu^{2}}+\log 4 \pi+\cdots\right)\right]
$$

as $\epsilon \rightarrow 0$.

It is easy to find counterterms for effective D3-brane Lagrangian (39). Their structure at the quartic level will be

$$
\left(s^{2}+t^{2}+u^{2}\right)\left(t_{8}\right) \mathrm{F}^{4} \rightarrow\left(t_{8}\right)_{\mu_{1} \nu_{1} \mu_{2} \nu_{2} \mu_{3} \nu_{3} \mu_{4} \nu_{4}} \partial_{\alpha} \mathrm{F}_{\mu_{1} \nu_{1}} \partial_{\alpha} \mathrm{F}_{\mu_{2} \nu_{2}} \partial_{\beta} \mathrm{F}_{\mu_{3} \nu_{3}} \partial_{\beta} \mathrm{F}_{\mu_{4} \nu_{4}},
$$

or, in the notations of [15] and [6], the counterterms are:

$$
T_{4}^{D 3}\left(\mathrm{~F}_{i j} \mathrm{~F}^{j k} \mathrm{~F}_{k l} \mathrm{~F}^{l i}-\frac{1}{4}\left(\mathrm{~F}_{i j} \mathrm{~F}_{j i}\right)^{2}\right)
$$

where

$$
T_{4}^{D 3}=-\frac{\alpha^{4}}{(4 \pi)^{2}} \frac{1}{16 \epsilon}\left(s^{2}+t^{2}+u^{2}\right)
$$

Using the relations (55)-(67) between the amplitudes with different particle content it is easy to find the corrections and the counterterms for all other four-particle interactions of $\mathcal{N}=4$ multiplets in this theory.

\section{Different $\kappa$-gauges}

The Killing gauge is obviously not the only choice for fixing the $\kappa$-gauge. In [3] it was proposed to use the gauge where $\theta_{1}=0$ and $\theta_{2}=\lambda$. Then the supersymmetry transformations will be:

$$
\begin{aligned}
\delta \bar{\theta} & =\bar{\epsilon}+\bar{\kappa}(1-\Gamma)+\xi^{\mu} \partial_{\mu} \bar{\theta} \\
\delta X^{m} & =\bar{\epsilon} \Gamma^{m} \theta-\bar{\kappa}(1-\Gamma) \Gamma^{m} \theta+\xi^{\mu} \partial_{\mu} X^{m} .
\end{aligned}
$$

where $\Gamma=\left(\begin{array}{cc}0 & \zeta \\ \tilde{\zeta} & 0\end{array}\right)$ was defined in (15).

The requirement $\delta \bar{\theta}_{1}=0$ and the static gauge condition $\delta X^{p}=0$ determine the connection between the $\kappa$ and $\epsilon$ parameters, so that the final supersymmetry 
transformations are [3]:

$$
\begin{aligned}
\delta_{\kappa+\varepsilon+\xi} \bar{\lambda}= & \bar{\epsilon}_{2}+\bar{\epsilon}_{1} \zeta+\xi^{\mu} \partial_{\mu} \bar{\lambda} \\
\delta_{\kappa+\varepsilon+\xi} y^{t}= & \left(\bar{\epsilon}_{2}-\bar{\epsilon}_{1} \zeta\right) \Gamma^{t} \lambda+\xi^{\mu} \partial_{\mu} y^{t} \\
\delta_{\kappa+\varepsilon+\xi} A_{\mu}= & \left(\bar{\epsilon}_{1} \zeta-\bar{\epsilon}_{2}\right)\left(\Gamma_{\mu}+\Gamma_{t} \partial_{\mu} y^{t}\right) \lambda \\
& +\left(\frac{1}{3} \bar{\epsilon}_{2}-\bar{\epsilon}_{1} \zeta\right) \Gamma_{m} \lambda \bar{\lambda} \Gamma^{m} \partial_{\mu} \lambda+\xi^{\rho} \partial_{\rho} A_{\mu}+\partial_{\mu} \xi^{\rho} A_{\rho} .
\end{aligned}
$$

The index $m$ is a $\mathrm{d}=10$ index, which includes both $\mu(p)$ and $t$ values $(t$ is a sixdimensional index for scalar fields). The induced world-volume metric in this case is:

$$
G_{\mu \nu}=\eta_{m n} L_{\mu}^{m} L_{\nu}^{n}, \quad L_{\mu}^{m}=\partial_{\mu} X^{m}-i \bar{\lambda} \Gamma^{m} \partial_{\mu} \lambda
$$

and

$$
\mathcal{F}_{\mu \nu}=F_{\mu \nu}-i \bar{\lambda}\left(\Gamma_{\mu}+\Gamma_{t} \partial_{\mu} y^{t}\right) \partial_{\nu} \lambda+i \bar{\lambda}\left(\Gamma_{\nu}+\Gamma_{t} \partial_{\nu} y^{t}\right) \partial_{\mu} \lambda
$$

The choice of $\kappa$-gauge affects only the part of the Lagrangian that contains fermions leaving the purely bosonic terms unchanged. In the $\theta_{1}=0$ gauge the fermionic part of the Lagrangian up to the quartic order in the fields is:

$$
\begin{aligned}
L_{\text {ferm }}^{(4)} & =i \bar{\theta} \not \partial \theta+i \alpha \bar{\theta} \Gamma^{t} \partial_{\mu} y^{t} \partial^{\mu} \theta+\frac{i \alpha}{2}\left(\bar{\theta} \Gamma^{\mu} \partial_{\nu} \theta-\mathrm{i} \bar{\theta} \Gamma^{\nu} \partial_{\mu} \theta\right) \mathrm{F}_{\mu \nu} \\
& +\frac{\alpha^{2}}{2}\left(\bar{\theta} \Gamma^{t} \partial_{\mu} \theta \bar{\theta} \Gamma^{t} \partial_{\mu} \theta-(\bar{\theta} \not \partial \theta)^{2}\right)-i \alpha^{2}\left(\partial_{\mu} y^{t} \partial_{\nu} y^{t} \bar{\theta} \Gamma^{\nu} \partial_{\mu} \theta-\frac{1}{2} \partial_{\mu} y^{t} \partial_{\mu} y^{t} \bar{\theta} \not \partial \theta\right) \\
& +\frac{i \alpha^{2}}{2}\left(\left(\bar{\theta} \Gamma^{\mu} \partial_{\nu} \theta+\bar{\theta} \Gamma^{\nu} \partial_{\mu} \theta\right) \mathrm{F}_{\mu \lambda} \mathrm{F}_{\lambda \nu}-\frac{1}{2} \bar{\theta} \not \partial \theta \mathrm{F}_{\mu \nu} \mathrm{F}_{\mu \nu}\right) \\
& +\frac{i \alpha^{2}}{2} \mathrm{~F}_{\mu \nu}\left(\bar{\theta} \Gamma^{t} \partial_{\mu} y^{t} \partial_{\nu} \theta-\bar{\theta} \Gamma^{t} \partial_{\nu} y^{t} \partial_{\mu} \theta\right) .
\end{aligned}
$$

In this gauge the Lagrangian contains the three-particles vertices along with the quartic terms. Four-particle tree amplitudes with fermions will contain a set of tree diagrams where line $m$ denotes both gauge $A_{\mu}$ and scalar $y^{t}$ bosons and — stands for the fermions $\theta$ :

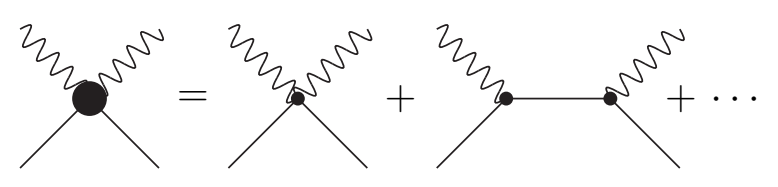




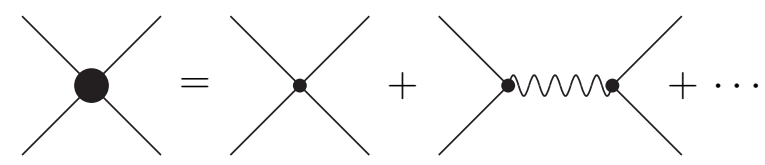

Calculations of these tree amplitudes show that they are the same for both cases of the $\kappa$-gauges. Both theories have the same one loop corrections and nontrivial counterterms in $\mathrm{d}=4$.

\section{Conclusion}

The Born-Infeld type actions have received a lot of attention in the last few years. This type of action plays a crucial role for the D3 brane theory. The rich structure of interaction terms in this theory makes it especially interesting to study them. The other interesting question that arises in this theory is a question about the form of supersymmetry transformations after the fixing of the $\kappa$-gauge.

We have presented the supersymmetry transformations, Feynman rules and oneloop corrections to the $\kappa$-gauge fixed D3 brane Born-Infeld theory up to the quartic order. We would like to stress here that the Feynman rules following from the gaugefixed supersymmetric Born-Infeld action turned out to be rather simple at least for the 4-point one loop calculations, which has allowed us to perform the quantum calculation in this very unusual theory.

Our calculations show that D3 brane theory in the flat IIB background has a nontrivial counterterm in $\mathrm{d}=4$. We have found that the structure of the counterterm as well as finite corrections includes terms like $(\partial \mathrm{F})^{4}$. We hope that our calculations may help to shed some light on the relations between the properties of the fundamental theory including the D3 branes and the quantum $\mathcal{N}=4$ supersymmetric YM gauge theory.

We also consider these calculations as a preparation for the studies of the more complicated theories, e.g. the D3 brane action in $A d S_{5} \times S^{5}$ background or, possibly, for a few interacting D3 branes with non-Abelian gauge field in the action. We have 
found that the use of the Killing gauge for $\kappa$-symmetry combined with the helicity amplitudes technique has simplified our calculations significantly. Hopefully these methods will be useful for calculations in some other class of interesting problems.

\section{Acknowledgments}

I am grateful to R. Kallosh for the help in the statement of the problem and for support and encouragement. I am thankful to L. Dixon for very useful discussions that helped me in my calculations. I am also thankful to J. Rahmfeld and M. Perelstein with whom we have discussed many issues in the D-brane theory and helicity amplitude formalism. I would also like to thank Michael Peskin and Arkady Tseytlin for the important comments on this paper.

This research was supported by the Department of Energy under grant DOEFG05-91ER40627 and partly under contract DE-AC03-76SF00515. This work was also supported in part by the NSF grant PHY-9870115.

\section{Appendix}

We use chiral representation for $\mathrm{d}=6$ dimensional $\gamma^{(6)}$ matrices following M. Sohnius notations [18]. In this representation $\gamma^{(6)}$ is written as

$$
\tilde{\gamma}^{t}=\left(\begin{array}{cc}
0 & \left(\tilde{\sigma}_{t}\right)_{I J} \\
\left(\tilde{\sigma}_{t}^{-1}\right)^{I J} & 0
\end{array}\right)
$$

where matrices $\left(\tilde{\sigma}_{t}\right)_{I J}$ satisfies the conditions:

$$
\begin{aligned}
\operatorname{tr} \tilde{\sigma}_{t} \tilde{\sigma}_{t^{\prime}}^{-1} & =4 \delta_{t t^{\prime}} \\
\left(\tilde{\sigma}_{t}^{-1}\right)^{I J} & =-\frac{1}{2} \epsilon^{I J K L}\left(\tilde{\sigma}_{t}\right)_{K L}
\end{aligned}
$$


A general $d=1032$ component complex spinor will be:

$$
\theta=\left(\begin{array}{c}
\lambda_{\alpha I} \\
\chi_{\alpha}^{I} \\
\bar{\omega}_{I}^{\dot{\alpha}} \\
\bar{\varphi}^{\dot{\alpha} I}
\end{array}\right), \quad \bar{\theta}=\left(\omega^{\alpha I}, \varphi_{I}^{\alpha}, \bar{\lambda}_{\dot{\alpha}}^{I}, \bar{\chi}_{\dot{\alpha} I}\right)
$$

The $\mathrm{d}=10$ Majorana and chirality conditions [18] are:

$$
\begin{aligned}
& \theta=C^{(10)} \bar{\theta}^{T} \quad, C^{(10)}=C^{(4)} \otimes C^{(6)}=\left(\begin{array}{cc}
-\epsilon_{\alpha \beta} & 0 \\
0 & -\epsilon^{\dot{\alpha} \dot{\beta}}
\end{array}\right) \otimes\left(\begin{array}{ll}
0 & 1 \\
1 & 0
\end{array}\right) \\
& \theta=\frac{1}{2}\left(1-\Gamma^{(11)}\right) \theta, \quad \Gamma^{(11)}=\gamma^{5} \otimes \gamma^{(7)}=i \gamma^{5} \otimes\left(\begin{array}{cc}
1 & 0 \\
0 & -1
\end{array}\right) .
\end{aligned}
$$

They will give the constraints on the spinors components:

$$
\begin{aligned}
\bar{\varphi}^{\dot{\alpha} I} & =\bar{\lambda}^{\dot{\alpha} I}=\left(\lambda_{\beta I}\right)^{\dagger} \epsilon^{\dot{\beta} \dot{\alpha}}, \quad \bar{\omega}_{I}^{\dot{\alpha}}=\bar{\chi}_{\dot{\alpha} I}=\left(\chi_{\beta}^{I}\right)^{\dagger} \epsilon^{\dot{\beta} \dot{\alpha}} \\
\bar{\omega}_{I}^{\dot{\alpha}} & =\chi_{\alpha}^{I}=0 .
\end{aligned}
$$

The surviving 16 components are

$$
\theta=\left(\begin{array}{c}
\lambda_{\alpha I} \\
0 \\
0 \\
\bar{\lambda}^{\dot{\alpha} I}
\end{array}\right) ; \quad \bar{\theta}=\left(0, \lambda_{I}^{\alpha}, \bar{\lambda}_{\dot{\alpha}}^{I}, 0\right) .
$$

The new scalar fields $s_{I J}$ with manifest $\mathrm{SU}(4)$ indexes $I, J=1, \cdots 4$ are

$$
s_{I J}=-\frac{1}{2}\left(\tilde{\sigma}_{t}\right)_{I J} y^{t} ; s^{I J}=\frac{1}{2}\left(\tilde{\sigma}_{t}^{-1}\right)^{I J} y^{t}
$$

and $y^{t} y^{t}=s^{I J} s_{I J}$.

\section{References}

[1] M.Cederwall, A. von Gussich, B.E.W. Nilsson, A. Westerber, The Dirichlet Super-Three-Brane in Ten-Dimensional Type IIB Supergravity, Nucl.Phys. B490 (1997) 163, hep-th/9610148. 
[2] E. Bergshoeff and P.K. Townsend, Super D-branes, Nucl. Phys. B490 (1997) 145 ,hep-th/9611173.

[3] M. Aganagic, C. Popescu and J.H. Schwarz, Gauge-invariant and and gauge-fixed D-brane actions, Nucl. Phys. B495 (1997) 99, hep-th/9612080.

[4] R. R. Metsaev and A. A. Tseytlin, Supersymmetric d3-brane action in $\operatorname{ads}(5) \mathrm{x}$ s(5), Phys. Lett. B436 (1998) 281 ,hep-th/9806095.

[5] S. Mandelstam, Nucl. Phys. B213 (1983) 149; L. Brink, O. Lindgren and B.E.W. Nilsson, Phys. Lett. 123B (1983) 323.

[6] N.Marcus and A, Sagnotti, Nucl. Phys. B256 (1985) 77.

[7] R. Kallosh, J. Rahmfeld, The GS String Action on ads(5) x s**5, Phys.Lett. B443 (1998) 143, hep-th/9808038.

[8] O.D. Andreev and A.A. Tseytlin, Nucl. Phys. B311 (1988/89) 205.

[9] A. Hashimoto and I. R. Klebanov, Decay of Excited D-branes,Phys. Lett. B381 (1996) 437, hep-th/9604065; Scattering of Strings from D-branes, Nucl. Phys. Proc. Suppl. 55B (1997) 118, hep-th/9611214.

[10] M.Mangano and S.J.Parke Phys.Rep. 200 (1991) 301.

[11] Z. Bern, L. Dixon and D.A. Kosower, Ann. Rev. Nucl. Part. Sci. 46 (1996) 109, hep-ph/9602280.

[12] F.A. Berends, R. Kleiss, P. De Causmaecker, R. Gastmans and T.T. Wu, Phys. Lett. 103B (1981) 124; P. De Causmaecker, R. Gastmans W. Troostand T.T. Wu, Nucl. Phys. B206 (1982) 124; R. Kleiss and W.J. Stirling, Nucl. Phys. B262 (1985) 235;J.F. Gunion and Z. Kunszt, Phys. Lett. 161B (1985) 333; Z. Xu, D.-H. Zhang and L. Chang, Nucl. Phys. B291 (1987) 392.

[13] M.T. Grisaru, H.N. Pendleton and P. van Nieuwenhuizen, Phys. Rev. D15 (1977) 996; M.T. Grisaru, H.N. Pendleton, Nucl. Phys. B124 (1977) 81; S.J. Parke and T. Taylor, Phys. Lett. 157B (1985) 81. 
[14] L.D. Landau, Nucl. Phys. 13 (1959) 181; S. Mandelstam, Phys. Rev. 112 (1958) 1344, 115 (1959) 1741; R. E. Cutkosky, J. Math. Phys. 1 (1960) 429.

[15] Z. Bern, L. Dixon, D.C. Dunbar, M. Perelstein, J.S. Rozowsky, On the Relationship between Yang-Mills Theory and Gravity and its Implication for Ultraviolet Divergences, Nucl.Phys. B530 (1998) 401, hep-th/9802162.

[16] J.Polchinsky, Phys. Rev. Lett. 75 (1995) 4724; TASI Lectures on D-Branes , hep-th/9611050; String Theory (Cambridge University Press, 1998).

[17] R. Kallosh, Worldvolume Supersymmetry, hep-th/9709069, Phys.Rev. D57 (1998) 3214.

[18] M.Sohnius, Phys.Rep. 128 (1985) 39.

[19] M.B. Green, J.H. Schwarz and E. Witten, Superstring Theory (Cambridge University Press, 1987)

[20] J.E. Paton and H.-M. Chan, Nucl. Phys. B10 (1969) 516; P. Cvitanovic, P.G. Lauwers and P.N. Scharabach, Nucl. Phys. B186 (1981) 165; F.A. Berends and W.T. Giele,Nucl.Phys. B294 (1987) 700; M. Mangano, S.J. Parke and Z. Xu, Nucl. Phys. B298 (1988) 653; M. Mangano, Nucl.Phys. B309 (1988) 461; D. Zeppenfeld, Int.J. Mod. Phys. A3 (1988) 2175.

[21] Z. Bern,J.S. Rozowsky and B. Yan, Phys. Lett. B401 (1997) hep-ph/9702424.

[22] Z. Bern, D.C. Dunbar, Nucl. Phys. B379 (1992) 562; A.G. Morgan, Phys. Lett. B351 (1995) 249, hep-ph/9502230.

[23] W.L. van Neerven, Nucl. Phys. B268 (1986) 453; Z. Bern, A.G. Morgan, Nucl. Phys. B467 (1996) 479.

[24] Z. Bern, L. Dixon,D.A. Kosower, Dimensionally Regulated Pentagon Integrals, Nucl.Phys. B412 (1994) 751, hep-ph/9306240. 